\title{
Distributed Reasoning with Fuzzy Description Logics
}

\author{
Jianjiang $\mathrm{Lu}^{1}$, Yanhui $\mathrm{Li}^{2}$, Bo Zhou ${ }^{1}$, Dazhou Kang ${ }^{2}$, and Yafei Zhang ${ }^{1}$ \\ ${ }^{1}$ Institute of Command Automation, PLA University of Science and Technology, \\ Nanjing 210007, China \\ 2 Department of Computer Science and Engineering, Southeast University, \\ Nanjing 210096, P.R. China \\ jjluaseu.edu.cn
}

\begin{abstract}
By the development of Semantic Web, increasing demands for vague and distributed information representation have triggered a mass of theoretical and applied researches of fuzzy and distributed ontologies, whose main logical infrastructures are fuzzy and distributed description logics. However, current solutions are proposed respectively on one of these two aspects. By integrating $\mathcal{E}$-connection into fuzzy description logics, this paper proposes a novel logical approach to couple both fuzzy and distributed features within description logics. The main contribution of these paper is to propose a discrete tableau algorithm to achieve reasoning within this new logical system.
\end{abstract}

Keywords: ontologies; $\mathcal{E}$-connection; discrete tableau algorithm.

\section{Introduction}

The Semantic Web stands for the idea of a future Web, in which information is given well-defined meaning, better enabling intelligent Web information processing [1]. In the Semantic Web, ontology is a crucial knowledge representation model to express a shared understanding of information between users and machines, and description logics (DLs for short) are often named as the logic infrastructure of ontologies [2]. Along with the evolvement from current Web to the Semantic Web, the management of ill-structured, ill-defined or imprecise information plays a more and more important role in applications of the Semantic Web, such as document retrieval [3], search engine [4] and query refinement [5]. This trend calls for ontologies with capability to deal with uncertainty. However, classical DLs are two-value-based languages. The need for expressing uncertainty in the Semantic Web has triggered extending classical DLs with fuzzy capabilities, yielding Fuzzy DLs (FDLs for short) [6,7,8,9]. Meanwhile, Working with multiple distributed ontologies brings a growing body of work in distributed research of description logic. In the distribution extension of classical DLs, Cuenca Grau et al integrated the $\mathcal{E}$-connections formalism [10] into OWL in a compact and natural way by defining "links" that stand for the inter-ontology relations [11]. Their extension is largely based on reasoning technique in classical DLs with general TBoxes.

The main difficulty in achieving similar distributed extension within FDLs and combining fuzzy and distributed features within DLs is that reasoning with general TBox in FDLs is still a hard problem. In this year, we propose a discrete tableau algorithm 
to solve this problem [12], that can be considered as a base technique to achieve distributed reasoning in FDLs. In this paper, we will extend our discretization algorithm in distributed case and combine $\mathcal{E}$-connections to propose a distributed extension of FDLs (here we focus on $\mathcal{F} \mathcal{S H \mathcal { N }}$ [8], a complex FDL with inverse role, role hierarchy and unqualified number restriction.) and a corresponding tableau reasoning algorithm within this extension, hence achieve distributed reasoning within multiple FDL KBs.

\section{$2 \mathcal{E}$-Connection Between Fuzzy Description Logics}

\subsection{Fuzzy Links Between Two Knowledge Bases}

Let $\mathcal{K}_{1}$ and $\mathcal{K}_{2}$ be two $\mathcal{F} \mathcal{S H \mathcal { I N }}$ KBs, $\mathcal{I}_{1}=\left\langle\Delta^{\mathcal{I}_{1}},{ }^{\mathcal{I}_{1}}\right\rangle$ and $\mathcal{I}_{2}=\left\langle\Delta^{\mathcal{I}_{2}},{ }^{\mathcal{I}_{2}}\right\rangle$ be their fuzzy interpretations. $\mathrm{E}_{12}$ is a set of fuzzy links (denoted $E_{12}$ and $F_{12}$ ) that connect these two $\mathcal{K}_{1}$ and $\mathcal{K}_{2}$. We define $\mathcal{I}_{12}=\left\langle\Delta^{\mathcal{I}_{12}},{ }^{\mathcal{I}_{12}}\right\rangle$ as the fuzzy interpretation of $E_{12}$, where $\Delta^{\mathcal{I}_{12}}=\Delta^{\mathcal{I}_{1}} \times \Delta^{\mathcal{I}_{2}}$ and for any $E_{12} \in \mathrm{E}_{12}$, ${ }^{\mathcal{I}_{12}}$ interprets it as a membership function: $\Delta^{\mathcal{I}_{1}} \times \Delta^{\mathcal{I}_{2}} \rightarrow[0,1]$. And to describe constraints among fuzzy links, we propose fuzzy link axioms: $E_{12} \sqsubseteq F_{12}$, where $E_{12}$ and $F_{12}$ are fuzzy links. A interpretation $\mathcal{I}_{12}$ satisfies the above fuzzy link axioms, iff for any $d \in \Delta^{\mathcal{I}_{1}}$ and any $d^{\prime} \in \Delta^{\mathcal{I}_{2}}, E_{12}^{\mathcal{I}_{12}}\left(d, d^{\prime}\right) \leq F_{12}^{\mathcal{I}_{12}}\left(d, d^{\prime}\right)$. An LBox $L_{12}$ is a finite set of fuzzy link axioms, $\mathcal{I}_{12}$ satisfies $L_{12}$, iff it satisfies every axiom in $L_{12}$.

These two FDL KBs $\mathcal{K}_{1}$ and $\mathcal{K}_{2}$ and their LBox $L_{12}$ construct a simple Combined Distributed FDL KB $\Sigma=\left(\mathcal{K}_{1}, \mathcal{K}_{2}, L_{12}\right)$. By introducing fuzzy link, we allow two new concepts $\mathcal{K}_{1}: \exists E_{12} . C_{2}$ and $\forall E_{12} . C_{2}$ in $\mathcal{K}_{1}$, where $E_{12}$ is a fuzzy link in $\mathcal{E}_{12}$ and $C_{2}$ is a fuzzy concept in $\mathcal{K}_{2}$. These two concepts are considered as normal fuzzy concepts in $\mathcal{K}_{1}$, hence they can appear in TBox and ABox of $\mathcal{K}_{1}$.

For example, let $\mathcal{K}_{1}$ and $\mathcal{K}_{2}$ be two KBs about animal and person respectively. $\operatorname{Dog}_{1}$ and Person 2 are fuzzy concepts in $\mathcal{K}_{1}$ and $\mathcal{K}_{2}$, and lovewith ${ }_{12}$ is a fuzzy link in $L_{12}$. By using fuzzy links, we can define a new fuzzy concept Friendlydog ${ }_{1}$ in $\mathcal{K}_{1}$ 's TBox:

$$
\text { Friendlydog }{ }_{1} \equiv \exists \text { lovewith }_{12} . \text { Person }_{2} \sqcap \operatorname{Dog}_{1}
$$

\subsection{Combined Distributed Fuzzy Description Logic Knowledge Bases}

In above subsection, we discuss the fuzzy links between two FDL KBs and give a simple example of Combined Distributed FDL (CDFDL for short) KBs. Now we will give a general definition of it.

Definition 1. a CDFDL KB is a pair $\Sigma=\left(\mathcal{K}_{S}, L_{S}\right)$, where $\mathcal{K}_{S}$ is a set of FDL KBs: $\mathcal{K}_{S}=\left\{\mathcal{K}_{1}, \ldots, \mathcal{K}_{m}\right\}$, and $L_{S}$ is a set of LBoxes that connect any two knowledge bases in $\mathcal{K}_{S}: L_{S}=\left\{L_{i j} \mid 1 \leq i, j \leq m\right.$ and $\left.i \neq j\right\}$. For any fuzzy concept $C_{j}$ in $\mathcal{K}_{j}$ and any fuzzy link $E_{i j}$ in $L_{i j}$ and, the following expressions are also considered as fuzzy concepts in $\mathcal{K}_{i}: \exists E_{i j} . C_{j}$ and $\forall E_{i j} . C_{j}$.

An interpretation of a CDFDL KB is a pair $\mathcal{I}=\left(\left\{\mathcal{I}_{i}\right\},\left\{\mathcal{I}_{i j}\right\}\right)$, where $\mathcal{I}_{i}$ is an interpretation of $\mathcal{K}_{i}$ and correspondingly $\mathcal{I}_{i j}$ is an interpretation of $L_{i j}$. For any fuzzy concept $C_{j}$ (role $R_{j}$ ) in $\mathcal{K}_{j}, C_{j}{ }^{\mathcal{I}}=C_{j}{ }^{\mathcal{I}_{j}}\left(R_{j}{ }^{\mathcal{I}}=R_{j}{ }^{\mathcal{I}_{j}}\right.$ ); for any fuzzy link $E_{i j}$ in $\mathcal{I}_{i j}$, 
$E_{i j}{ }^{\mathcal{I}}=E_{i j}{ }^{\mathcal{I}_{i j}}$; for any individual $a_{j}$ in $\mathcal{K}_{j}, a_{j}{ }^{\mathcal{I}}=a_{j}{ }^{\mathcal{I}_{j}}$; and for $\exists E_{i j} \cdot C_{j}$ and $\forall E_{i j} \cdot C_{j}$, their interpretation are inductively defined as:

$$
\begin{aligned}
& \exists E_{i j} \cdot C_{j}^{\mathcal{I}}(d)=\sup _{d^{\prime} \in \Delta^{\mathcal{I}_{i}}}\left\{\min \left(E_{i j}{ }^{\mathcal{I}_{i j}}\left(d, d^{\prime}\right), C_{j}^{\mathcal{I}_{j}}\left(d^{\prime}\right)\right)\right\} \\
& \forall E_{i j} \cdot C_{j}^{\mathcal{I}}(d)=\inf _{d^{\prime} \in \Delta^{\mathcal{I}_{i}}}\left\{\max \left(1-E_{i j}{ }^{\mathcal{I}_{i j}}\left(d, d^{\prime}\right), C_{j}^{\mathcal{I}_{j}}\left(d^{\prime}\right)\right)\right\}
\end{aligned}
$$

An interpretation $\mathcal{I}$ is a model of $\Sigma=\left(\mathcal{K}_{S}, L_{S}\right)$, iff $\mathcal{I}$ satisfies every $\mathcal{K}_{i}$ in $\mathcal{K}_{S}$ and every $L_{i j}$ in $L_{S}$. In this paper, we will propose a discrete tableau algorithm to decide satisfiability of CDFDL KBs $\Sigma$, which is based on the semantical discretization technique discussed in the following section.

\section{Semantical Discretization}

In this section, we will propose a novel semantical discretization technique to achieve such translations: if a CDFDL KB has a fuzzy model, we use the discretization to translate it into a special model, in which any value of membership degree functions belongs to a given discrete degree set $S$ and its cardinality $|S|$ is polynomial of the sum of the cardinality $\left|\mathcal{A}_{i}\right|$ of the $\mathrm{ABox} \mathcal{A}_{i}$ in every $\mathrm{KB} \mathcal{K}_{i}$. And we call it a discrete model within $S$.

The main issue in semantical discretization is to decide the discrete degree set $S$. Let us now proceed formally in the creation of $S$. Given $\Sigma=\left(\mathcal{K}_{S}=\left\{\mathcal{K}_{1}, \ldots, \mathcal{K}_{m}\right\}, L_{S}=\right.$ $\left.\left\{L_{i j} \mid 1 \leq i, j \leq m, i \neq j\right\}\right)$, and $\mathcal{K}_{i}=\left\langle\mathcal{T}_{i}, \mathcal{R}_{i}, \mathcal{A}_{i}\right\rangle$. Let $N_{d}$ be the set of degrees appearing in any ABox: $N_{d}=\left\{n \mid \alpha \bowtie n \in \mathcal{A}_{i}, 1 \leq i \leq m\right\}$. From $N_{d}$, we define the degree closure $N_{d}^{*}=\{0,0.5,1\} \cup N_{d} \cup\left\{n \mid 1-n \in N_{d}\right\}$ and order degrees in ascending order: $N_{d}^{*}=\left\{n_{0}, n_{1}, \ldots, n_{s}\right\}$, where for any $0 \leq i \leq s, n_{i}<n_{i+1}$. For any two backto-back elements $n_{i}, n_{i+1} \in N_{d}^{*}$, we insert their median $m_{i+1}=\left(n_{i}+n_{i+1}\right) / 2$ to get $S=\left\{n_{0}, m_{1}, n_{1}, \ldots, n_{s-1}, m_{s}, n_{s}\right\}$. We call $S$ a discrete degree set w.r.t $\Sigma$. Obviously for any $1 \leq i \leq s, m_{i}+m_{s+1-i}=1$ and $n_{i-1}<m_{i}<n_{i}$. Note that:

$$
|S|=2 s+1=\mathrm{O}\left(\left|N_{d}\right|\right)=\mathrm{O}\left(\sum_{i=1}^{m}\left|\mathcal{A}_{i}\right|\right) .
$$

Lemma 1. For any $\mathcal{K}_{i}=\left\langle\mathcal{T}_{i}, \mathcal{R}_{i}, \mathcal{A}_{i}\right\rangle$ and any discrete degree set $S$ w.r.t $\Sigma$, if $\mathcal{K}_{i}$ has a fuzzy model, it has a discrete model within $S$.

The proof of this lemma is an extension of the proof in FDL cases [12]. Meanwhile, to verify the soundness of our discretization, we have the following lemma.

Lemma 2. For any $L_{i j}$ and any discrete degree set $S$ w.r.t $\Sigma$, if $L_{i j}$ has a fuzzy model, it has a discrete model within $S$.

Since a discrete model is also a fuzzy model of $\Sigma$, we get the following theorem to guarantee the equivalence between existence of fuzzy models and discrete models.

Theorem 1. For any $\Sigma=\left(\mathcal{K}_{S}=\left\{\mathcal{K}_{1} \ldots \mathcal{K}_{m}\right\}, L_{S}=\left\{L_{i j}\right\}\right)$ and any discrete degree set $S$ w.r.t $\Sigma, \Sigma$ has a fuzzy model iff it has a discrete model within $S$. 


\section{Discrete Tableau Algorithm}

Before expressing discrete tableau algorithms formally, here we introduce some notations. It will be assumed that the concepts appearing in tableau algorithms are written in NNF [13]. The set of subconcepts of a concept $C$ is denoted as $\operatorname{sub}(C)$. For a CDFDL KB $\Sigma$, we define $\operatorname{sub}\left(\mathcal{K}_{i}\right)$ as the union of all $\operatorname{sub}(C)$, for any concept $C$ appears in $\mathcal{K}_{i}$. And we use the symbols $\triangleright$ and $\triangleleft$ as two placeholders for the inequalities $\geq,>$ and $\leq$, $<$, and the symbols $\bowtie^{-}, \triangleright^{-}$and $\triangleleft^{-}$to denote their reflections, for example, $\geq$and $\leq$ are reflections to each other. Finally we define $\langle\bowtie, n\rangle$ as a degree pair. Two degree pairs are called conjugated, iff they satisfy the following conditions (see table 1).

Table 1. Conjugated pairs

\begin{tabular}{|c|c|c|}
\hline & $\langle<, m\rangle$ & $\langle\leq, m\rangle$ \\
\hline$\langle\geq, n\rangle$ & $n \geq m$ & $n>m$ \\
\hline$\langle>, n\rangle$ & $\neg \exists n_{1} \in S$ with $n<n_{1}<m$ & $n \geq m$ \\
\hline
\end{tabular}

Now we define the discrete tableau for $\Sigma$. Let $\mathrm{R}_{\mathcal{K}_{i}}$ and $\mathrm{O}_{\mathcal{K}_{i}}$ be the sets of roles and individuals appearing in $\mathcal{K}_{i}$. A discrete tableau $\mathrm{T}$ for $\Sigma$ within a degree set $S$ is a pair: $\mathrm{T}=\left\langle\left\{\mathrm{T}_{i}\right\},\left\{\mathcal{E}_{i j}\right\}\right\rangle, \mathrm{T}_{i}=\left\langle\mathcal{O}_{i}, \mathcal{L}_{i}, \mathcal{E}_{i}, \mathcal{V}_{i}\right\rangle, 1 \leq i, j \leq m$ and $i \neq j$, where

- $\mathcal{O}_{i}$ : a nonempty set of nodes;

- $\mathcal{L}_{i}: \mathcal{O}_{i} \rightarrow 2^{M_{i}}, M_{i}=\operatorname{sub}\left(\mathcal{K}_{i}\right) \times\{\geq,>, \leq,<\} \times S$

- $\mathcal{E}_{i}: \mathrm{R}_{\mathcal{K}_{i}} \rightarrow 2^{Q_{i}}, Q_{i}=\left\{\mathcal{O}_{i} \times \mathcal{O}_{i}\right\} \times\{\geq,>, \leq,<\} \times S$

- $\mathcal{V}_{i}: \mathrm{O}_{\mathcal{K}_{i}} \rightarrow \mathcal{O}_{i}$, maps any individual into a corresponding node in $\mathcal{O}_{i}$.

- $\mathcal{E}_{i j}: \mathrm{E}_{i j} \rightarrow 2^{Q_{i j}}, Q_{i j}=\left\{\mathcal{O}_{i} \times \mathcal{O}_{j}\right\} \times\{\geq,>, \leq,<\} \times S$;

Any $\mathrm{T}_{i}$ has a forest-like structure, which is a collection of trees that correspond to individuals in the ABox $\mathcal{A}_{i}$. Every tree consists of nodes standing for the individuals, and edges representing the relations between two nodes (individuals). Each node $d$ is labelled with a set $\mathcal{L}(d)$ of degree triples: $\langle C, \bowtie, n\rangle$, which denotes the membership degree of $d$ being an instance of $C \bowtie n$. A pair of triple $\langle C, \bowtie, n\rangle$ and $\left\langle C, \bowtie^{-}, m\right\rangle$ are conjugated if $\langle\bowtie, n\rangle$ and $\left\langle\bowtie^{-}, m\right\rangle$ are conjugated. In any $\mathrm{T}_{i}$, for any $d, d^{\prime} \in \mathcal{O}_{i}$, $a, b \in \mathrm{O}_{\mathcal{K}_{i}}, C, D \in \operatorname{sub}\left(\mathcal{K}_{i}\right)$ and $R \in \mathrm{R}_{\mathcal{K}_{i}}$, the following conditions must hold:

1. There does not exist two conjugated degree triples in $\mathcal{L}_{i}(d)$;

2. There does not exist mistake triples: $\langle\perp, \geq, n\rangle(n>0),\langle\top, \leq, n\rangle(n<1),\langle\perp$, $, n\rangle,\langle\top,<, n\rangle,\langle C,>, 1\rangle$ and $\langle C,<, 0\rangle$ in $\mathcal{L}_{i}(d)$;

3. If $C \sqsubseteq D \in \mathcal{T}$, then there must be some $n \in S$ with $\langle C, \leq, n\rangle$ and $\langle D, \geq, n\rangle$ in $\mathcal{L}_{i}(d)$

4. If $\langle C, \bowtie, n\rangle \in \mathcal{L}_{i}(d)$, then $\left\langle\operatorname{nnf}(\neg C), \bowtie^{-}, 1-n\right\rangle \in \mathcal{L}_{i}(d)$;

5. If $\langle C \sqcap D, \triangleright, n\rangle \in \mathcal{L}_{i}(d)$, then $\langle C, \triangleright, n\rangle$ and $\langle D, \triangleright, n\rangle \in \mathcal{L}_{i}(d)$;

6. If $\langle C \sqcap D, \triangleleft, n\rangle \in \mathcal{L}_{i}(d)$, then $\langle C, \triangleleft, n\rangle$ or $\langle D, \triangleleft, n\rangle \in \mathcal{L}_{i}(d)$;

7. If $\langle C \sqcup D, \triangleright, n\rangle \in \mathcal{L}_{i}(d)$, then $\langle C, \triangleright, n\rangle$ or $\langle D, \triangleright, n\rangle \in \mathcal{L}_{i}(d)$;

8. If $\langle C \sqcup D, \triangleleft, n\rangle \in \mathcal{L}_{i}(d)$, then $\langle C, \triangleleft, n\rangle$ and $\langle D, \triangleleft, n\rangle \in \mathcal{L}_{i}(d)$; 
9. If $\langle\forall R . C, \triangleright, n\rangle \in \mathcal{L}_{i}(d),\left\langle\left\langle d, d^{\prime}\right\rangle, \triangleright^{\prime}, m\right\rangle \in \mathcal{E}_{i}(R)$, and $\left\langle\triangleright^{\prime}, m\right\rangle$ is conjugated with $\left\langle\triangleright^{-}, 1-n\right\rangle$, then $\langle C, \triangleright, n\rangle \in \mathcal{L}_{i}\left(d^{\prime}\right)$;

10. If $\langle\forall R . C, \triangleleft, n\rangle \in \mathcal{L}_{i}(d)$, then there must be a node $d^{\prime} \in \mathcal{O}_{i}$ with $\left\langle\left\langle d, d^{\prime}\right\rangle, \triangleleft^{-}, 1-\right.$ $n\rangle \in \mathcal{E}_{i}(R)$ and $\langle C, \triangleleft, n\rangle \in \mathcal{L}_{i}\left(d^{\prime}\right)$;

11. If $\langle\exists R . C, \triangleright, n\rangle \in \mathcal{L}_{i}(d)$, then there must be a node $d^{\prime} \in \mathcal{O}_{i}$ with $\left\langle\left\langle d, d^{\prime}\right\rangle, \triangleright, n\right\rangle \in$ $\mathcal{E}_{i}(R)$ and $\langle C, \triangleright, n\rangle \in \mathcal{L}_{i}\left(d^{\prime}\right)$;

12. If $\langle\exists R . C, \triangleleft, n\rangle \in \mathcal{L}_{i}(d),\left\langle\left\langle d, d^{\prime}\right\rangle, \triangleright^{\prime}, m\right\rangle \in \mathcal{E}_{i}(R)$, and $\left\langle\triangleright^{\prime}, m\right\rangle$ is conjugated with $\langle\triangleleft, n\rangle$, then $\langle C, \triangleleft, n\rangle \in \mathcal{L}_{i}\left(d^{\prime}\right)$;

13. If $\langle\forall P . C, \triangleright, n\rangle \in \mathcal{L}_{i}(d),\left\langle\left\langle d, d^{\prime}\right\rangle, \triangleright^{\prime}, m\right\rangle \in \mathcal{E}_{i}(R)$ for some $R \sqsubseteq^{*} P$ with Trans $(R)=$ true and $\left\langle\triangleright^{\prime}, m\right\rangle$ is conjugated with $\left\langle\triangleright^{-}, 1-n\right\rangle$, then $\langle\forall R . C, \triangleright, n\rangle \in \mathcal{L}_{i}\left(d^{\prime}\right)$;

14. If $\langle\exists P . C, \triangleleft, n\rangle \in \mathcal{L}_{i}(d),\left\langle\left\langle d, d^{\prime}\right\rangle, \triangleright^{\prime}, m\right\rangle \in \mathcal{E}_{i}(R)$ for some $R \sqsubseteq^{*} P$ with Trans $(R)=$ true and $\left\langle\triangleright^{\prime}, m\right\rangle$ is conjugated with $\langle\triangleleft, n\rangle$, then $\langle\exists R . C, \triangleleft, n\rangle \in \mathcal{L}_{i}\left(d^{\prime}\right)$;

15. If $\langle\geq p R, \triangleright, n\rangle \in \mathcal{L}_{i}(d)$, then $\mid\left\{d^{\prime}\left|\left\langle\left\langle d, d^{\prime}\right\rangle, \triangleright, n\right\rangle \in \mathcal{E}_{i}(R)\right|\right\} \geq p$;

16. If $\langle\geq p R, \triangleleft, n\rangle \in \mathcal{L}_{i}(d)$, then $\left|\left\{d^{\prime} \mid\left\langle\left\langle d, d^{\prime}\right\rangle, \triangleright^{\prime}, m\right\rangle \in \mathcal{E}_{i}(R)\right\}\right|\left\langle p\right.$, where $\left\langle\triangleright^{\prime}, m\right\rangle$ is conjugated with $\langle\triangleleft, n\rangle$;

17. If $\langle\leq p R, \triangleright, n\rangle \in \mathcal{L}_{i}(d)$, then $\left|\left\{d^{\prime} \mid\left\langle\left\langle d, d^{\prime}\right\rangle, \triangleright^{\prime}, m\right\rangle \in \mathcal{E}_{i}(R)\right\}\right|<p+1$, where $\left\langle\triangleright^{\prime}, m\right\rangle$ is conjugated with $\left\langle\triangleright^{-}, 1-n\right\rangle$;

18. If $\langle\leq p R, \triangleleft, n\rangle \in \mathcal{L}_{i}(d),\left|\left\{d^{\prime} \mid\left\langle\left\langle d, d^{\prime}\right\rangle, \triangleleft^{-}, 1-n\right\rangle \in \mathcal{E}_{i}(R)\right\}\right| \geq p+1$;

19. If $\left\langle\left\langle d, d^{\prime}\right\rangle, \bowtie, n\right\rangle \in \mathcal{E}_{i}(R)$, then $\left\langle\left\langle d^{\prime}, d\right\rangle, \bowtie, n\right\rangle \in \mathcal{E}_{i}(\operatorname{Inv}(R))$;

20. If $\left\langle\left\langle d, d^{\prime}\right\rangle, \triangleright, n\right\rangle \in \mathcal{E}_{i}(R)$ and $R \sqsubseteq^{*} P$, then $\left\langle\left\langle d, d^{\prime}\right\rangle, \triangleright, n\right\rangle \in \mathcal{E}_{i}(P)$;

21. If $a: C \bowtie n \in \mathcal{A}_{i}$, then $\langle C, \bowtie, n\rangle \in \mathcal{L}_{i}\left(\mathcal{V}_{i}(a)\right)$;

22. If $\langle a, b\rangle: R \bowtie n \in \mathcal{A}_{i}$, then $\left\langle\left\langle\mathcal{V}_{i}(a), \mathcal{V}_{i}(b)\right\rangle, \bowtie, n\right\rangle \in \mathcal{E}_{i}(R)$;

23. If $a \neq b \in \mathcal{A}_{i}$, then $\mathcal{V}_{i}(a) \neq \mathcal{V}_{i}(b)$.

From conditions 1-2, the discrete tableau contains no clash. Condition 3 deals with general TBoxes: for any $C \sqsubseteq D \in \mathcal{T}$, we adopt a direct extension of reasoning technique in DLs: since any membership degree value in the discrete models belongs to $S$, for any node $d$, we guess $d: C=n$ and $d: D=m$, for some $n, m \in S$ and $n \leq m$. Then we add $\langle C, \leq, n\rangle$ and $\langle D, \geq, n\rangle$ in $\mathcal{L}(d)$. Conditions 4-20 are necessary for the soundness of discrete tableaus. Conditions 21-23 ensure the correctness of individual mapping function $\mathcal{V}()$.

Additionally, we add some constraints to deal with fuzzy links. For any $d \in \mathcal{O}_{i}$, $d^{\prime} \in \mathcal{O}_{j}, E_{i j}, F_{i j} \in L_{i j}$ and $C_{j} \in \operatorname{sub}\left(\mathcal{K}_{j}\right)$, the following conditions must hold:

24. If $\left\langle\left\langle d, d^{\prime}\right\rangle, \triangleright, n\right\rangle \in \mathcal{E}_{i j}\left(E_{i j}\right)$ and $E_{i j} \sqsubseteq F_{i j} \in L_{i j}$, then $\left\langle\left\langle d, d^{\prime}\right\rangle, \triangleright, n\right\rangle \in \mathcal{E}_{i j}\left(F_{i j}\right)$;

25. If $\left\langle\forall E_{i j} . C_{j}, \triangleright, n\right\rangle \in \mathcal{L}_{i}(d),\left\langle\left\langle d, d^{\prime}\right\rangle, \triangleright^{\prime}, m\right\rangle \in \mathcal{E}_{i j}\left(E_{i j}\right)$, and $\left\langle\triangleright^{\prime}, m\right\rangle$ is conjugated with $\left\langle\triangleright^{-}, 1-n\right\rangle$, then $\langle C, \triangleright, n\rangle \in \mathcal{L}_{j}\left(d^{\prime}\right)$;

26. If $\left\langle\forall E_{i j} . C_{j}, \triangleleft, n\right\rangle \in \mathcal{L}_{i}(d)$, then there must be a node $d^{\prime} \in \mathcal{O}_{j}$ with $\left\langle\left\langle d, d^{\prime}\right\rangle, \triangleleft^{-}, 1\right.$ $-n\rangle \in \mathcal{E}_{i j}\left(E_{i j}\right)$ and $\langle C, \triangleleft, n\rangle \in \mathcal{L}_{j}\left(d^{\prime}\right)$;

27. If $\left\langle\exists E_{i j} . C_{j}, \triangleright, n\right\rangle \in \mathcal{L}_{i}(d)$, then there must be a node $d^{\prime} \in \mathcal{O}_{j}$ with $\left\langle\left\langle d, d^{\prime}\right\rangle, \triangleright, n\right\rangle$ $\in \mathcal{E}_{i j}\left(E_{i j}\right)$ and $\langle C, \triangleright, n\rangle \in \mathcal{L}_{j}\left(d^{\prime}\right)$;

28. If $\left\langle\exists E_{i j} . C_{j}, \triangleleft, n\right\rangle \in \mathcal{L}_{i}(d),\left\langle\left\langle d, d^{\prime}\right\rangle, \triangleright^{\prime}, m\right\rangle \in \mathcal{E}_{i j}\left(E_{i j}\right)$, and $\left\langle\triangleright^{\prime}, m\right\rangle$ is conjugated with $\langle\triangleleft, n\rangle$, then $\langle C, \triangleleft, n\rangle \in \mathcal{L}_{j}\left(d^{\prime}\right)$; 
Condition 24 guarantees that tableau satisfies the restriction of LBoxes. Conditions 2528 are distributed extensions of classical conditions to deal with $\forall$ and $\exists$ restriction.

Theorem 2. For any $\Sigma=\left(\mathcal{K}_{S}=\left\{\mathcal{K}_{1} \ldots \mathcal{K}_{m}\right\}, L_{S}=\left\{L_{i j}\right\}\right)$ and any discrete degree set $S$ w.r.t $\Sigma, \Sigma$ has a discrete model within $S$ iff it has a discrete tableau $\mathrm{T}$ within $S$.

From theorem 1 and 2, an algorithm that constructs a discrete tableau of $\Sigma$ within $S$ can be considered as a decision procedure for the satisfiability of $\Sigma$. The discrete tableau algorithm works on a completion forest $\mathrm{F}_{\Sigma}$ with a set $S \neq$ to denote " $\neq$ " relation between nodes and a tag function $W()$ : for any node $x, W(x)$ denotes that $x$ is an individual in the $W(x)$-th $\mathrm{KB}$. When $W(x)=W(y)=i, x$ is labelled with $\mathcal{L}_{i}(x) \subseteq$ $M_{i}=\operatorname{sub}\left(\mathcal{K}_{i}\right) \times\{\geq,>, \leq,<\} \times S$; and the edge $\langle x, y\rangle$ is labelled $\mathcal{L}_{i}(\langle x, y\rangle)=\{\langle R, \bowtie$ , $n\rangle\}$, for some $R \in \mathrm{R}_{\mathcal{K}_{i}}$ and $n \in S$. When $W(x)=i \neq W(y)=j$, the edge $\langle x, y\rangle$ is labelled $\mathcal{L}_{i j}(\langle x, y\rangle)=\{\langle E, \bowtie, n\rangle\}$, for some $E \in \mathrm{E}_{i j}$ and $n \in S$. The tableau algorithm initializes $\mathrm{F}_{\mathcal{K}}$ to contain a root node $x_{a}$ for each individual $a$ in any $\mathrm{O}_{\mathcal{K}_{i}}$, sets $W\left(x_{a}\right)=i$ and labels $x_{a}$ with $\mathcal{L}_{i}\left(x_{a}\right)=\left\{\langle C, \bowtie, n\rangle \mid a: C \bowtie n \in \mathcal{A}_{i}\right\}$. Moreover, for any pair $\left\langle x_{a}, x_{b}\right\rangle, \mathcal{L}_{i}\left\langle x_{a}, x_{b}\right\rangle=\left\{\langle R, \bowtie, n\rangle \mid\langle a, b\rangle: R \bowtie n \in \mathcal{A}_{i}\right\}$, and for any $a \neq b \in \mathcal{A}_{i}$, we add $\left\langle x_{a}, x_{b}\right\rangle \in S^{\neq}$. The algorithm expands the forest $\mathrm{F}_{\Sigma}$ either by extending $\mathcal{L}_{i}(x)$ for the current node $x$ or by adding new leaf node $y$ with expansion rules in table 2 .

In table 2, we adopt a optimized way to reduce " $\triangleleft$ rules": for any triple $\langle C, \triangleleft, n\rangle \in$ $\mathcal{L}_{i}(x)$ with " $\triangleleft$ ", we use $\neg^{\bowtie}$ rules to add its equivalence $\left\langle\operatorname{nnf}(C), \triangleleft^{-}, 1-n\right\rangle$ to $\mathcal{L}_{i}(x)$, and then deal it with $\triangleright$ rules. Edges are added when expanding $\langle\exists G . C, \triangleright, n\rangle$, $\langle\geq p G, \triangleright, n\rangle$ in $\mathcal{L}_{i}(x)$, where $G$ can be a fuzzy role or fuzzy link. A node $y$ is called an $G$-successor of another node $x$ and $x$ is called a $G$-predecessor of $y$, if $\langle G, \bowtie$ $, n\rangle \in \mathcal{L}_{i(i j)}(\langle x, y\rangle)$. Ancestor is the transitive closure of predecessor. And for any two connected nodes $x$ and $y$, we define $D_{G}(x, y)=\left\{\langle\bowtie, n\rangle \mid P \sqsubseteq^{*} G,\langle P, \bowtie, n\rangle \in\right.$ $\mathcal{L}_{i(i j)}(\langle x, y\rangle)$ or $\left.\langle\operatorname{Inv}(P), \bowtie, n\rangle \in \mathcal{L}_{i(i j)}(\langle y, x\rangle)\right\}$. If $D_{G}(x, y) \neq \emptyset, y$ is called a $R$ neighbor of $x$. As inverse role and number restriction are allowed in $\mathcal{S H I N}$, we make use of pairwise blocking technique [14] to ensure the termination and correctness of our tableau algorithm: a node $x$ is directly blocked by its ancestor $y$ iff (1) $x$ is not a root node; (2) $x$ and $y$ have predecessors $x^{\prime}$ and $y^{\prime}$, such that $\mathcal{L}_{i}(x)=\mathcal{L}_{i}(y)$ and $\mathcal{L}_{i}\left(x^{\prime}\right)=\mathcal{L}_{i}\left(y^{\prime}\right)$ and $\mathcal{L}_{i(i j)}\left(\left\langle y^{\prime}, y\right\rangle\right)=\mathcal{L}_{i(i j)}\left(\left\langle x^{\prime}, x\right\rangle\right)$. A node $\mathrm{x}$ is indirectly blocked if its predecessor is blocked. A node $\mathrm{x}$ is blocked iff it is either directly or indirectly blocked.

A completion forest $\mathrm{F}_{\mathcal{K}}$ is said to contain a clash, if for a node $x$ in $\mathrm{F}_{\mathcal{K}}$ with $W(x)=$ $i,(1) \mathcal{L}_{i}(x)$ contains two conjugated triples, or a mistake triple (see condition 2 in discrete tableau restriction); or (2) $\langle\geq p R, \triangleleft, n\rangle$ or $\left\langle\leq(p-1) R, \triangleleft^{-}, 1-n\right\rangle \in \mathcal{L}_{i}(x)$, and there are $p$ nodes $y_{1}, y_{2}, \ldots y_{p}$ in $\mathrm{F}_{\Sigma}$ : for any $1 \leq k \leq p,\left\langle R, \triangleright_{k}, m_{k}\right\rangle \in \mathcal{L}_{i}\left(\left\langle x, y_{k}\right\rangle\right)$, $\left\langle\triangleright_{k}, m_{k}\right\rangle$ is conjugated with $\langle\triangleleft, n\rangle$ and for any two nodes $y_{k}$ and $y_{q},\left\langle y_{k}, y_{q}\right\rangle \in S^{\neq}$. A completion forest $\mathrm{F}_{\Sigma}$ is clash-free if it does not contain a clash, and it is complete if none of the expansion rules are applicable. From pairwise blocking technique, the worst-case complexity of our tableau algorithm is 2NEXPTIME [15]. And the soundness and completeness of our tableau algorithm are guaranteed by the following theorem. 
Table 2. Expansion rules of discrete Tableau

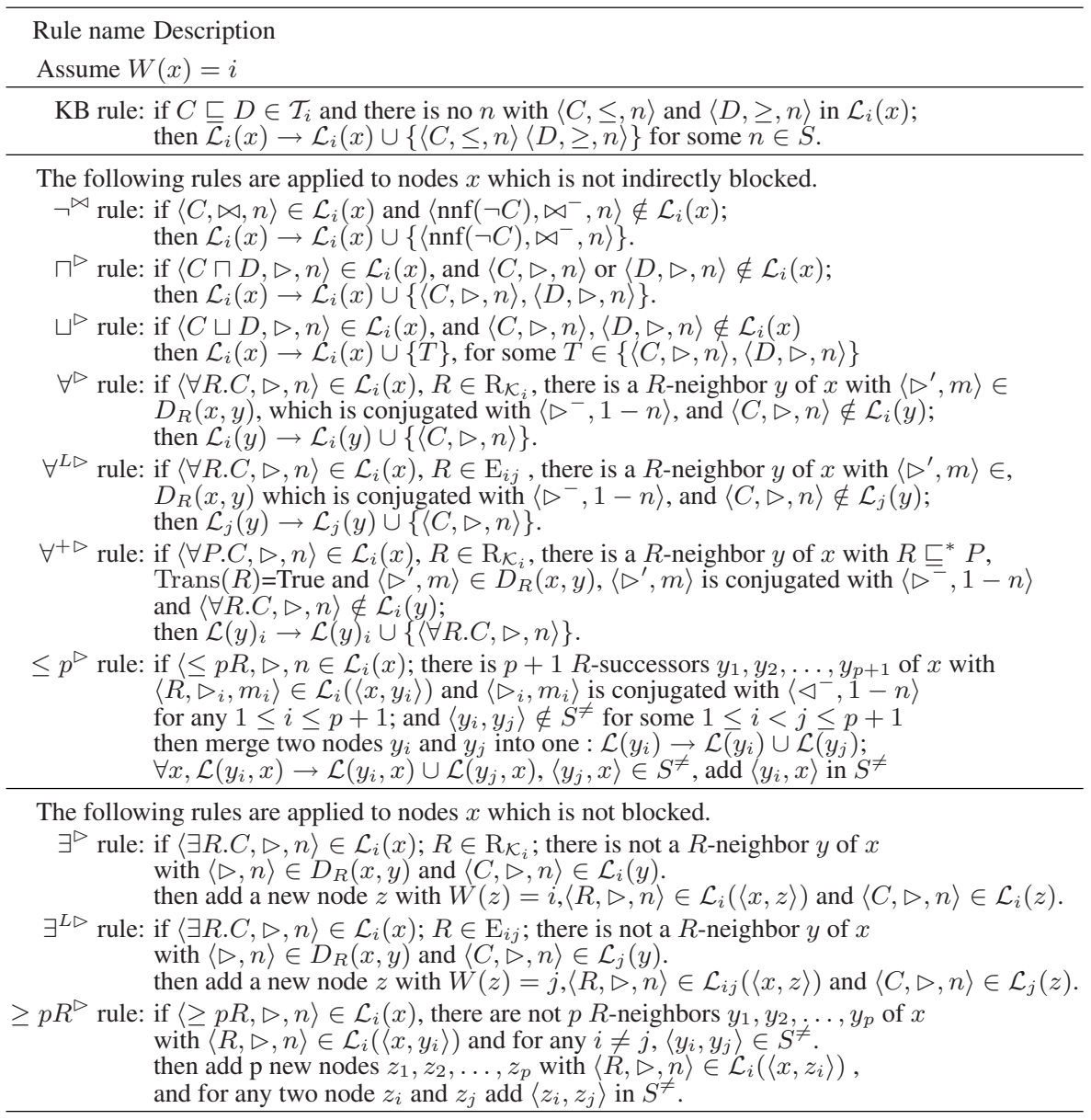

Theorem 3. For any $\Sigma=\left(\mathcal{K}_{S}=\left\{\mathcal{K}_{1} \ldots \mathcal{K}_{m}\right\}, L_{S}=\left\{L_{i j}\right\}\right)$ and any discrete degree set $S$ w.r.t $\Sigma$ has a discrete tableau within $S$ iff the tableau algorithm can construct a complete and clash-free completion forest.

\section{Conclusion}

By integrating $\mathcal{E}$-connection into FDLs, this paper proposes a novel logical approach to couple both fuzzy and distributed features within DLs. To achieve reasoning support within this new logical form CDFDL, we extend our semantical discretization in distributed case and design a discrete tableau reasoning algorithm. Our work can be considered as a logical foundation to support reasoning with multiple distributed fuzzy ontologies. 


\section{References}

1. Berners-Lee, T., Hendler, J., Lassila, O.: The semantic web. Scientific American 284 (2001) 34-43

2. Horrocks, I., Patel-Schneider, P.: Reducing owl entailment to description logic satisfiability. In: Proceeedings of the International Workshop on Description Logics (DL-05). (2003) 1-8

3. Parry, D. Fuzzy Logic and the Semantic Web. In: A fuzzy ontology for medical document retrieval. Elsevier Science, Oxford, UK (2006)

4. Widyantoro, D.H., Yen, J.: A fuzzy ontology-based abstract search engine and its user studies. In: FUZZ-IEEE. (2001) 1291-1294

5. Widyantoro, D., Yen, J.: Using fuzzy ontology for query refinement in a personalized abstract search engine. In: Proceedings of Joint 9th IFSA World Congress and 20th NAFIPS International Conference, Vancouver, Canada (2001)

6. Straccia, U.: A fuzzy description logic. In: Proceedings of AAAI-98, 15th National Conference on Artificial Intelligence, Madison, Wisconsin (1998) 594-599

7. Stoilos, G., Stamou, G., Tzouvaras, V., Pan, J., Horrocks, I.: Fuzzy owl: Uncertainty and the semantic web. In: Proceedings of International Workshop of OWL: Experiences and Directions, Galway (2005)

8. Stoilos, G., Stamou, G., Tzouvaras, V., Pan, J., Horrocks, I.: The fuzzy description logic shin. In: Proceedings of International Workshop of OWL: Experiences and Directions, Galway (2005)

9. Stoilos, G., Stamou, G., Tzouvaras, V., Pan, J., Horrock, I.: A Fuzzy Description Logic for Multimedia Knowledge Representation. In: Proc. of the International Workshop on Multimedia and the Semantic Web. (2005)

10. Kutz, O., Lutz, C., Wolter, F., Zakharyaschev, M.: E-connections of abstract description systems. Artificial Intelligence 156 (2004) 1-73

11. Cuenca Grau, B., Parsia, B., Sirin, E.: Working with multiple ontologies on the semantic web. In: Proceedings of the 3thrd International Semantic Web Conference. (2004)

12. Li, Y.H., Xu, B.W., Lu, J.J., Kang, D.Z.: Discrete tableaus for fshi. In: Proceedings of 2006 International Workshop on Description Logics - DL2006, The Lake District of the UK (2006)

13. Baader, F., Sattler, U.: An overview of tableau algorithms for description logics. Studia Logica 69 (2001) 5-40

14. Horrocks, I., Sattler, U.: A description logic with transitive and inverse roles and role hierarchies. Journal of Logic and Computation 9 (1999) 385-410

15. Horrocks, I., Sattler, U., Tobies, S.: Practical reasoning for expressive description logics. In: Proceedings of of LPAR99. (1999) 